\title{
ON THE TIME VALUE OF ABSOLUTE RUIN WITH DEBIT INTEREST
}

\author{
JUN CAI,* University of Waterloo
}

\begin{abstract}
Assume that the surplus of an insurer follows a compound Poisson surplus process. When the surplus is below zero or the insurer is on deficit, the insurer could borrow money at a debit interest rate to pay claims. Meanwhile, the insurer will repay the debts from her premium income. The negative surplus may return to a positive level. However, when the negative surplus is below a certain critical level, the surplus is no longer able to be positive. Absolute ruin occurs at this moment. In this paper, we study absolute ruin questions by defining an expected discounted penalty function at absolute ruin. The function includes the absolute ruin probability, the Laplace transform of the time to absolute ruin, the deficit at absolute ruin, the surplus just before absolute ruin, and many other quantities related to absolute ruin. First, we derive a system of integro-differential equations satisfied by the function and obtain a defective renewal equation that links the integro-differential equations in the system. Second, we show that when the initial surplus goes to infinity, the absolute ruin probability and the classical ruin probability are asymptotically equal for heavy-tailed claims while the ratio of the absolute ruin probability to the classical ruin probability goes to a positive constant that is less than one for light-tailed claims. Finally, we give explicit expressions for the function for exponential claims.
\end{abstract}

Keywords: Compound Poisson process; defective renewal equation; key renewal theorem; absolute ruin; deficit at absolute ruin; surplus just before absolute ruin; Gerber-Shiu function; debit interest; subexponential distribution; long-tailed distribution

2000 Mathematics Subject Classification: Primary 91B30

Secondary 60K05; $91 \mathrm{~B} 70$

\section{Introduction}

Let $U(t)=u+c t-\sum_{n=1}^{N(t)} X_{n}, t \geq 0$, be the compound Poisson surplus process for an insurer, where $u \geq 0$ is the initial surplus, $c>0$ is the premium rate, $\{N(t), t \geq 0\}$ is a Poisson process (with Poisson rate $\lambda>0$ ) denoting the number of claims up to time $t$, and $\left\{X_{n}, n \geq 1\right\}$ (representing the sizes of claims and independent of $\{N(t), t \geq 0\}$ ) is a sequence of independent and identically distributed nonnegative random variables with a common distribution function $F(x)$ that satisfies $F(0)=0$ and has a positive mean $\mu=\int_{0}^{\infty} \bar{F}(x) \mathrm{d} x>0$. Here, $\bar{F}(x)=$ $1-F(x)$ is the survival function of the distribution function $F(x)$.

We assume that when the surplus is negative or the insurer is on deficit, the insurer could borrow an amount of money equal to the deficit at a debit interest force $\delta>0$, or, equivalently, at a debit interest rate $\mathrm{e}^{\delta}-1>0$. Meanwhile, the insurer will repay the debts continuously from her premium income. Thus, the surplus of the insurer is driven under the debit interest force $\delta$ when the surplus is negative. The negative surplus may return to a positive level. However, when the negative surplus attains the level $-c / \delta$ or is below $-c / \delta$, the surplus is no longer

Received 28 September 2006; revision received 5 March 2007.

* Postal address: Department of Statistics and Actuarial Science, University of Waterloo, Waterloo, Ontario, Canada N2L 3G1. Email address: jcai@ math.uwaterloo.ca 
able to be positive, because the debts of the insurer at this time are greater than or equal to $c / \delta$, which is the present value at that time for all premium income available after that point. Absolute ruin occurs at this moment.

Absolute ruin has attracted attention in the literature. The absolute ruin probability with exponential claims has been studied by Dassios and Embrechts (1989) using the martingale approach. Embrechts and Schmidli (1994) discussed the absolute ruin probability in a general insurance risk model using the theory of piecewise deterministic Markov processes. Furthermore, Dickson and Egídio dos Reis (1997) considered the effect of interest on the negative surplus. In addition, the effect of interest on the positive surplus has been studied extensively in the literature. See, for example, Asmussen (2000), Cai (2004), Cai and Dickson (2002), Sundt and Teugels (1995), and many others. However, many questions related to absolute ruin have not yet been solved, such as the behavior of the absolute ruin probability with heavy-tailed claims, the relationship between the absolute ruin probability and the classical ruin probability, the Laplace transform of the time to absolute ruin, the deficit at absolute ruin, the surplus just before absolute ruin, and so on. In this paper, we study absolute ruin questions by defining an expected discounted penalty function at absolute ruin. The function includes the absolute ruin probability, the Laplace transform of the time to absolute ruin, the deficit at absolute ruin, the surplus just before absolute ruin, and many other quantities related to absolute ruin. It allows us to study the effect of debit interest on the negative surplus in a unified approach.

We denote the surplus of the insurer at time $t$ with the debit interest force $\delta$ by $U_{\delta}(t)$. This is the solution to

$$
\mathrm{d} U_{\delta}(t)=\left(c+\delta U_{\delta}(t) \mathbf{1}_{\left\{U_{\delta}(t)<0\right\}}\right) \mathrm{d} t-\mathrm{d} Y(t), \quad U_{\delta}(0)=u,
$$

where $Y_{t}=\sum_{i=1}^{N(t)} X_{i}$ and $\mathbf{1}_{\{C\}}$ means the indicator function of an event $C$. Furthermore, we denote the absolute ruin time of the surplus process $\left\{U_{\delta}(t), t \geq 0\right\}$ by $T_{\delta}$. Now, $T_{\delta}=$ $\inf \left\{t \geq 0: U_{\delta}(t) \leq-c / \delta\right\}$ and $T_{\delta}=\infty$ if $U_{\delta}(t)>-c / \delta$ for all $t \geq 0$. Thus, $\left|U_{\delta}\left(T_{\delta}\right)\right|$ is the deficit at absolute ruin. Furthermore, let $U_{\delta}\left(T_{\delta}^{-}\right)$denote the surplus just before absolute ruin. Notice that the deficit at absolute ruin is at least $c / \delta$ and the surplus just before absolute ruin could be in the range of $(-c / \delta, \infty)$. Thus, we define the expected discounted penalty function at absolute ruin by

$$
\Phi(u)=\mathrm{E}\left(\mathrm{e}^{-\alpha T_{\delta}} w\left(U_{\delta}\left(T_{\delta}^{-}\right),\left|U_{\delta}\left(T_{\delta}\right)\right|\right) \mathbf{1}_{\left\{T_{\delta}<\infty\right\}} \mid U_{\delta}(0)=u\right),
$$

where $w\left(x_{1}, x_{2}\right), x_{1}>-c / \delta, x_{2} \geq c / \delta$, is a nonnegative function which denotes the penalty due at absolute ruin, $u>-c / \delta$ is the initial surplus, and $\alpha \geq 0$ can be viewed as the argument for the Laplace transform of $T_{\delta}$ or an interest force for the calculation of the present value of the penalty. In particular, the absolute ruin probability, denoted by

$$
\psi(u)=\mathrm{P}\left(T_{\delta}<\infty \mid U_{\delta}(0)=u\right),
$$

satisfies $\psi(u)=\Phi(u)$ when $\alpha=0$ and $w\left(x_{1}, x_{2}\right)=1$ in $\Phi(u)$.

The function (1.1) is the counterpart of the expected discounted penalty function at ruin or the Gerber-Shiu function at ruin in the compound Poisson surplus process $\{U(t), t \geq 0\}$, which was introduced by Gerber and Shiu (1997), (1998). The Gerber-Shiu function has become a standard method used to study ruin theory in different risk models.

We point out that $\Phi(u)$ has different sample paths for $u \geq 0$ and $-c / \delta<u<0$. Hence, we distinguish the two situations by writing $\Phi(u)=\Phi_{+}(u)$ for $u \geq 0$ and $\Phi(u)=\Phi_{-}(u)$ for $-c / \delta<u<0$. Similarly, we write $\psi(u)=\psi_{+}(u)$ for $u \geq 0$ and $\psi(u)=\psi_{-}(u)$ 
for $-c / \delta<u<0$. In addition, we denote the ruin time of the compound Poisson surplus process $\{U(t), t \geq 0\}$ by $T$ and the classical ruin probability in the compound Poisson surplus process $\{U(t), t \geq 0\}$ by $\phi(u)$. Now, $T=\inf \{t \geq 0: U(t)<0\}$ and $T=\infty$ if $U(t) \geq 0$ for all $t \geq 0$. Furthermore, $\phi(u)=\mathrm{P}(T<\infty \mid U(0)=u)$.

In this paper, we assume that the safety loading factor defined by $\theta=c /(\lambda \mu)-1$ is positive. Obviously, $T \leq T_{\delta}$ and $\psi_{+}(u) \leq \phi(u)$ for $u \geq 0$. Under the assumption of the positive safety loading factor, we have $\lim _{u \rightarrow \infty} \psi_{+}(u)=0$ and $0<\psi_{+}(u) \leq \phi(u)<1$ for $u \geq 0$. Also, we assume that $\lim _{u \rightarrow \infty} \Phi_{+}(u)=0$. This holds naturally when $w\left(x_{1}, x_{2}\right)$ is a bounded function.

The rest of the paper is organized as follows. In Section 2, we derive a system of integrodifferential equations for $\Phi_{+}(u)$ and $\Phi_{-}(u)$ and determine boundary conditions for $\Phi_{+}(u)$ and $\Phi_{-}(u)$. In Section 3, we obtain a defective renewal equation for $\Phi_{+}(u)$ when $\alpha=0$. This case includes the absolute ruin probability, the deficit at absolute ruin, the surplus just before absolute ruin, the claim amount causing absolute ruin, and many others. The defective renewal equation links the integro-differential equations in the system. Based on the defective renewal equation, in Section 4 we show that when the initial surplus goes to infinity, the absolute ruin probability $\psi_{+}(u)$ and the classical ruin probability $\phi(u)$ are asymptotically equal for heavy-tailed claims while the ratio of the absolute ruin probability $\psi_{+}(u)$ to the classical ruin probability $\phi(u)$ goes to a positive constant that is less than one for light-tailed claims. Finally, in Section 5 we give explicit expressions for $\Phi_{+}(u)$ and $\Phi_{-}(u)$ for exponential claims.

\section{Integral and integro-differential equations for $\Phi_{+}(u)$ and $\Phi_{-}(u)$}

First, we derive the integral equations for $\Phi_{+}(u)$ and $\Phi_{-}(u)$ in the following theorem.

Theorem 2.1. When $u \geq 0$, we have

$$
\begin{aligned}
\Phi_{+}(u)= & \frac{\lambda}{c} \mathrm{e}^{(\lambda+\alpha) u / c} \int_{u}^{\infty} \mathrm{e}^{-(\lambda+\alpha) x / c} \\
& \times\left[\int_{0}^{x} \Phi_{+}(x-y) \mathrm{d} F(y)+\int_{x}^{x+c / \delta} \Phi_{-}(x-y) \mathrm{d} F(y)+A(x)\right] \mathrm{d} x,
\end{aligned}
$$

and, when $-c / \delta<u<0$, we have

$$
\begin{gathered}
\Phi_{-}(u)=\lambda(\delta u+c)^{(\lambda+\alpha) / \delta}\left\{\int_{u}^{0}(\delta x+c)^{-1-(\lambda+\alpha) / \delta}\left[\int_{0}^{x+c / \delta} \Phi_{-}(x-y) \mathrm{d} F(y)+A(x)\right] \mathrm{d} x\right. \\
+c^{-1-(\lambda+\alpha) / \delta} \int_{0}^{\infty} \mathrm{e}^{-(\lambda+\alpha) z / c} \\
\times\left[\int_{0}^{z} \Phi_{+}(z-y) \mathrm{d} F(y)\right. \\
\left.\left.+\int_{z}^{z+c / \delta} \Phi_{-}(z-y) \mathrm{d} F(y)+A(z)\right] \mathrm{d} z\right\},
\end{gathered}
$$

where the function $A(x)$ is given by

$$
A(x)=\int_{x+c / \delta}^{\infty} w(x, y-x) \mathrm{d} F(y) .
$$


Proof. By conditioning on the time and amount of the first claim and discounting the expected values to time 0 at the interest force $\alpha$, when $u \geq 0$ we obtain

$$
\begin{aligned}
\Phi_{+}(u)=\int_{0}^{\infty} \lambda \mathrm{e}^{-(\lambda+\alpha) t}[ & \int_{0}^{u+c t} \Phi_{+}(u+c t-y) \mathrm{d} F(y)+\int_{u+c t}^{u+c t+c / \delta} \Phi_{-}(u+c t-y) \mathrm{d} F(y) \\
& \left.+\int_{u+c t+c / \delta}^{\infty} w(u+c t, y-(u+c t)) \mathrm{d} F(y)\right] \mathrm{d} t
\end{aligned}
$$

Equation (2.4) for $\Phi_{+}(u)$ involves $\Phi_{-}(u)$ for $-c / \delta<u<0$. When the initial surplus is negative, the surplus is driven under the debit interest force $\delta$ before the surplus returns to the level zero. Thus, for $-c / \delta<u<0$, let $t_{0}=t_{0}(u)$ be the solution to $h_{\delta}(t, u)=$ $u \mathrm{e}^{\delta t}+c\left(\mathrm{e}^{\delta t}-1\right) / \delta=0$, namely $t_{0}=t_{0}(u)=\log (c /(c+\delta u))^{1 / \delta}$, which is the time when the surplus returns to the level zero if no claim occurs prior to time $t_{0}$. Furthermore, $h_{\delta}(t, u)<0$ for $t<t_{0}$ and $h_{\delta}\left(t_{0}, u\right)=0$. Moreover, $h_{\delta}(t, u)$ is the surplus at time $t \leq t_{0}$ if no claim occurs prior to time $t_{0}$. Thus, by conditioning on the time and amount of the first claim and discounting the expected values to time 0 at the interest force $\alpha$, when $-c / \delta<u<0$ we obtain

$$
\begin{aligned}
\Phi_{-}(u)=\int_{0}^{t_{0}} \lambda \mathrm{e}^{-(\lambda+\alpha) t}[ & \int_{0}^{h_{\delta}(t, u)+c / \delta} \Phi_{-}\left(h_{\delta}(t, u)-y\right) \mathrm{d} F(y) \\
& \left.+\int_{h_{\delta}(t, u)+c / \delta}^{\infty} w\left(h_{\delta}(t, u), y-h_{\delta}(t, u)\right) \mathrm{d} F(y)\right] \mathrm{d} t \\
+\int_{t_{0}}^{\infty} \lambda \mathrm{e}^{-(\lambda+\alpha) t} & {\left[\int_{0}^{c\left(t-t_{0}\right)} \Phi_{+}\left(c\left(t-t_{0}\right)-y\right) \mathrm{d} F(y)\right.} \\
& +\int_{c\left(t-t_{0}\right)}^{c\left(t-t_{0}\right)+c / \delta} \Phi_{-}\left(c\left(t-t_{0}\right)-y\right) \mathrm{d} F(y) \\
& \left.+\int_{c\left(t-t_{0}\right)+c / \delta}^{\infty} w\left(c\left(t-t_{0}\right), y-c\left(t-t_{0}\right)\right) \mathrm{d} F(y)\right] \mathrm{d} t .
\end{aligned}
$$

Now, changing variables $x=u+c t$ in (2.4), we obtain (2.1). Moreover, first changing variables $x=h_{\delta}(t, u)$ in the integrals with respect to $t$ from 0 to $t_{0}$ in (2.5), and then changing variables $z=c\left(t-t_{0}\right)$ in the integrals with respect to $t$ from $t_{0}$ to $\infty$ in (2.5), we obtain (2.2).

Remark 2.1. We point out that the integral equations (2.1) and (2.2) allow us to consider the differentiability of the functions $\Phi_{+}(u)$ and $\Phi_{-}(u)$. For instance, it is easy to see from (2.1) and (2.2) that $\Phi_{+}(u)$ and $\Phi_{-}(u)$ are differentiable on $(0, \infty)$ and $(-c / \delta, 0)$ respectively. Furthermore, they satisfy the following condition:

$$
\Phi_{+}(0)=\Phi_{-}(0-) \text {. }
$$

Based on (2.2), we can determine boundary conditions for $\Phi_{-}(u)$.

Proposition 2.1. If

$$
\lim _{u \downarrow-c / \delta} \int_{u}^{0}(\delta x+c)^{-1-(\lambda+\alpha) / \delta} A(x) \mathrm{d} x=\infty,
$$

then

$$
\lim _{u \downarrow-c / \delta} \Phi_{-}(u)=\frac{\lambda}{\lambda+\alpha} A\left(-\frac{c}{\delta}\right) .
$$


If

$$
\lim _{u \downarrow-c / \delta} \int_{u}^{0}(\delta x+c)^{-1-(\lambda+\alpha) / \delta} A(x) \mathrm{d} x<\infty,
$$

then

$$
\lim _{u \downarrow-c / \delta} \Phi_{-}(u)=0 .
$$

Proof. From (2.2), we notice that if

$$
\lim _{u \downarrow-c / \delta} \int_{u}^{0}\left[(\delta x+c)^{-1-(\lambda+\alpha) / \delta} \int_{0}^{x+c / \delta} \Phi_{-}(x-y) \mathrm{d} F(y)\right] \mathrm{d} x<\infty,
$$

then

$$
\lim _{u \downarrow-c / \delta} \lambda(\delta u+c)^{(\lambda+\alpha) / \delta} \int_{u}^{0}\left[(\delta x+c)^{-1-(\lambda+\alpha) / \delta} \int_{0}^{x+c / \delta} \Phi_{-}(x-y) \mathrm{d} F(y)\right] \mathrm{d} x=0 .
$$

Furthermore, if

$$
\lim _{u \downarrow-c / \delta} \int_{u}^{0}\left[(\delta x+c)^{-1-(\lambda+\alpha) / \delta} \int_{0}^{x+c / \delta} \Phi_{-}(x-y) \mathrm{d} F(y)\right] \mathrm{d} x=\infty,
$$

then l'Hôpital's rule yields

$$
\lim _{u \downarrow-c / \delta} \lambda(\delta u+c)^{(\lambda+\alpha) / \delta} \int_{u}^{0}\left[(\delta x+c)^{-1-(\lambda+\alpha) / \delta} \int_{0}^{x+c / \delta} \Phi_{-}(x-y) \mathrm{d} F(y)\right] \mathrm{d} x=0 .
$$

Hence, (2.11) always holds. Thus, letting $u \rightarrow-c / \delta$ in (2.2) yields

$$
\lim _{u \downarrow-c / \delta} \Phi_{-}(u)=\lim _{u \downarrow-c / \delta} \lambda(\delta u+c)^{(\lambda+\alpha) / \delta} \int_{u}^{0}(\delta x+c)^{-1-(\lambda+\alpha) / \delta} A(x) \mathrm{d} x .
$$

Therefore, if (2.7) holds, by l'Hôpital's rule and (2.12) we obtain (2.8). Furthermore, if (2.9) holds, then (2.12) yields (2.10).

As we will see in Section 5, the boundary conditions given in Proposition 2.1 are necessary when we solve the integro-differential equations or differential equations satisfied by $\Phi_{-}(u)$ to obtain explicit expressions for $\Phi_{-}(u)$.

Now, by differentiating the integral equations (2.1) and (2.2), we obtain the integro-differential equations for $\Phi_{+}(u)$ and $\Phi_{-}(u)$ in the following theorem.

Theorem 2.2. When $u \geq 0$, we have

$$
\Phi_{+}^{\prime}(u)=\frac{\lambda+\alpha}{c} \Phi_{+}(u)-\frac{\lambda}{c}\left[\int_{0}^{u} \Phi_{+}(u-y) \mathrm{d} F(y)+B(u)\right],
$$

and, when $-c / \delta<u<0$, we have

$$
\Phi_{-}^{\prime}(u)=\frac{\lambda+\alpha}{\delta u+c} \Phi_{-}(u)-\frac{\lambda}{\delta u+c}\left[\int_{0}^{u+c / \delta} \Phi_{-}(u-y) \mathrm{d} F(y)+A(u)\right],
$$

where

$$
B(u)=\int_{u}^{u+c / \delta} \Phi_{-}(u-y) \mathrm{d} F(y)+A(u) .
$$

In addition, the boundary conditions for $\Phi_{+}(u)$ and $\Phi_{-}(u)$ are given by (2.6), Proposition 2.1, and $\lim _{u \rightarrow \infty} \Phi_{+}(u)=0$. 
We remark that the equation (2.14) for $\Phi_{-}(u)$ is independent of $\Phi_{+}(u)$. However, $\Phi_{-}(u)$ is subject to the boundary condition (2.6) which is involved with $\Phi_{+}(u)$. Furthermore, it is easy to see from (2.6), (2.13), and (2.14) that $\Phi_{+}(u)$ and $\Phi_{-}(u)$ satisfy

$$
\Phi_{+}^{\prime}(0)=\Phi_{-}^{\prime}(0-) \text {. }
$$

\section{The link between $\Phi_{+}(u)$ and $\Phi_{-}(u)$ via a defective renewal equation}

When $\alpha=0$, we derive a defective renewal equation for $\Phi_{+}(u)$ in the following theorem.

Theorem 3.1. When $\alpha=0, \Phi_{+}(u)$ satisfies the defective renewal equation

$$
\Phi_{+}(u)=\frac{\lambda}{c} \int_{u}^{\infty} B(t) \mathrm{d} t+\frac{1}{1+\theta} \int_{0}^{u} \Phi_{+}(u-y) \mathrm{d} F_{\mathrm{e}}(y), \quad u \geq 0,
$$

where $F_{\mathrm{e}}(y)=\int_{0}^{y} \bar{F}(x) \mathrm{d} x / \mu, y \geq 0$, is the equilibrium distribution function of $F(x)$ and $\theta=c /(\lambda \mu)-1>0$ is the safety loading factor. In particular, for any $u \geq 0$ we have

$$
\Phi_{+}(u)=\frac{1+\theta}{\theta} \int_{0}^{u} z(u-x) \mathrm{d} G(x),
$$

where

$$
z(x)=\frac{\lambda}{c} \int_{x}^{\infty} B(t) \mathrm{d} t
$$

and

$$
G(x)=\sum_{n=0}^{\infty} \frac{\theta}{1+\theta}\left(\frac{1}{1+\theta}\right)^{n} F_{\mathrm{e}}^{(n)}(x)
$$

is a compound geometric distribution function. Here, $F_{\mathrm{e}}^{(n)}$ is the $n$-fold convolution of $F_{\mathrm{e}}$ with itself.

Proof. Replacing $u$ by $t$ in (2.13), integrating both sides of the equation from 0 to $u$ with respect to $t$, and performing integration by parts, for $u \geq 0$ we obtain

$$
\Phi_{+}(u)=\Phi_{+}(0)-\frac{\lambda}{c} \int_{0}^{u} B(t) \mathrm{d} t+\frac{\lambda \mu}{c} \int_{0}^{u} \Phi_{+}(u-y) \mathrm{d} F_{\mathrm{e}}(y)+\frac{\alpha}{c} \int_{0}^{u} \Phi_{+}(t) \mathrm{d} t .
$$

When $\alpha=0,(3.4)$ is reduced to

$$
\Phi_{+}(u)=\Phi_{+}(0)-\frac{\lambda}{c} \int_{0}^{u} B(t) \mathrm{d} t+\frac{\lambda \mu}{c} \int_{0}^{u} \Phi_{+}(u-y) \mathrm{d} F_{\mathrm{e}}(y) .
$$

By the boundary condition $\lim _{u \rightarrow \infty} \Phi_{+}(u)=0$, the dominated convergence theorem, and letting $u \rightarrow \infty$ in (3.5), we obtain

$$
\Phi_{+}(0)=\frac{\lambda}{c} \int_{0}^{\infty} B(t) \mathrm{d} t .
$$

Thus, substituting (3.6) into (3.5) yields

$$
\Phi_{+}(u)=\frac{\lambda}{c} \int_{0}^{\infty} B(t) \mathrm{d} t-\frac{\lambda}{c} \int_{0}^{u} B(t) \mathrm{d} t+\frac{\lambda \mu}{c} \int_{0}^{u} \Phi_{+}(u-y) \mathrm{d} F_{\mathrm{e}}(y),
$$

which implies (3.1). Equation (3.1) is a defective renewal equation. Hence, by Theorem 2.1 of Resnick (1992), we immediately obtain (3.2). 
Note that the expression (3.2) of $\Phi_{+}(u)$ depends on $B(u)$ which is a function of $\Phi_{-}(u)$. Once $\Phi_{-}(u)$ is obtained, the expression for $\Phi_{+}(u)$ is given by (3.2). Furthermore, (3.1) allows us to discuss the properties of $\Phi_{+}(u)$ based on those for defective renewal equations. As applications of Theorem 3.1, in Section 4 we derive asymptotical formulae for the absolute ruin probability when claims are heavy-tailed and light-tailed. To do so, we recall that the classical ruin probability $\phi(u)$ is given by

$$
\phi(u)=\frac{1}{\theta} \int_{0}^{u} \bar{F}_{\mathrm{e}}(u-x) \mathrm{d} G(x), \quad u \geq 0 .
$$

\section{Asymptotic results for heavy-tailed and light-tailed claims}

A claim size distribution is said to be heavy-tailed if the moment generating function of the distribution does not exist. We first recall some classes of heavy-tailed distributions that will be used in this section. A distribution $F$ is said to be subexponential, written $F \in \&$, if, for any $n \geq 2, \lim _{x \rightarrow \infty} \bar{F}^{(n)}(x) / \bar{F}(x)=n$, where $\bar{F}^{(n)}(x)=1-F^{(n)}(x)$. A distribution $F$ is said to be long-tailed, written $F \in \mathcal{L}$, if, for any $y>0, \lim _{x \rightarrow \infty} \bar{F}(x-y) / \bar{F}(x)=1$, or, equivalently, for any $y>0, \lim _{x \rightarrow \infty} \bar{F}(x+y) / \bar{F}(x)=1$. It is well known that $\delta \subset \mathcal{L}$. Throughout this paper, $a(u) \sim b(u)$ means $a(u) / b(u) \rightarrow 1$ as $u \rightarrow \infty$.

Lemma 4.1. Consider a defective renewal equation

$$
Z(x)=v(x)+\beta \int_{0}^{x} Z(x-y) \mathrm{d} F(y), \quad x \geq 0,
$$

where $0<\beta<1, F(x)$ is a distribution function supported on $[0, \infty)$, and $v(x)$ is a locally bounded function defined on $[0, \infty)$. Assume that $v(x) \geq 0$ is right-continuous and decreasing with $\lim _{x \rightarrow \infty} v(x)=0$. If $F \in \delta, W \in \delta$, and $\sup _{x \geq 0}\{\overline{\bar{F}}(x) / \bar{W}(x)\}<\infty$, then

$$
Z(x) \sim \frac{v(x)}{1-\beta},
$$

where $W(x)=(v(0)-v(x)) / v(0)=1-\bar{W}(x)$ is a distribution function supported on $[0, \infty)$.

Proof. The proof is given in Theorem 3.1(i) of Yin and Zhao (2006), which is based on Proposition 1.1 (c) of Cai and Tang (2004) and other results about heavy-tailed distributions in the class 8 .

Theorem 4.1. Let $f(x)$ be the density function of the distribution function $F(x)$ and $r(x)=$ $f(x) / \bar{F}(x)$ be the failure rate function. If $r(x) \rightarrow 0$ as $x \rightarrow \infty$ and $F_{\mathrm{e}} \in \&$, then

$$
\psi_{+}(u) \sim \frac{1}{\theta} \bar{F}_{\mathrm{e}}(u)
$$

Proof. For $w\left(x_{1}, x_{2}\right)=1$ and $\alpha=0$, we have $\Phi_{-}(u)=\psi_{-}(u)$ and $A(u)=\bar{F}(u+c / \delta)$. Thus, by (2.15) we obtain

$$
\frac{B(u)}{\bar{F}(u)}=\int_{-c / \delta}^{0} \psi_{-}(t) r(u-t) \frac{\bar{F}(u-t)}{\bar{F}(u)} \mathrm{d} t+\frac{\bar{F}(u+c / \delta)}{\bar{F}(u)} .
$$

Note that $0<\psi_{-}(t)<1$ and $0 \leq \bar{F}(u-t) / \bar{F}(u) \leq 1$ for any $-c / \delta<t<0$. Obviously, $r(x) \rightarrow 0$ as $x \rightarrow \infty$ implies that $r(x)$ is bounded on $\left[x_{0}, \infty\right)$ for some constant $x_{0}>0$. 
Furthermore, as pointed out in the paragraph after Proposition 2 of Embrechts and Villasenor (1988), $r(x) \rightarrow 0$ as $x \rightarrow \infty$ implies that $F \in \mathcal{L}$. Also see Bingham et al. (1987, p. 12).

Thus, by the dominated convergence theorem and $F \in \mathcal{L}$, we know that the first term in (4.2) goes to zero and the second term in (4.2) goes to one as $u \rightarrow \infty$. Hence, $\lim _{u \rightarrow \infty} B(u) / \bar{F}(u)=1$, which, together with l'Hôpital's rule and (3.3), implies that

$$
\lim _{u \rightarrow \infty} \frac{z(u)}{\bar{F}_{\mathrm{e}}(u)}=\frac{\lambda \mu}{c} \lim _{u \rightarrow \infty} \frac{B(u)}{\bar{F}(u)}=\frac{\lambda \mu}{c}=\frac{1}{1+\theta} .
$$

Therefore, the survival function $\bar{H}(x)$ defined by $\bar{H}(x)=z(x) / z(0)=1-H(x)$ satisfies

$$
\lim _{u \rightarrow \infty} \frac{\bar{H}(u)}{\bar{F}_{\mathrm{e}}(u)}=\frac{1}{(1+\theta) z(0)},
$$

which means that the distribution $H \in \delta$ because $F_{\mathrm{e}} \in \delta$ and the class $\delta$ is preserved under tailequivalence. Note that both $\bar{H}(x)$ and $\bar{F}_{\mathrm{e}}(x)$ are continuous on $(0, \infty)$ with $\bar{H}(0)=\bar{F}_{\mathrm{e}}(0)=1$. Thus, (4.4) implies that $\sup _{x \geq 0}\left\{\bar{H}(x) / \bar{F}_{\mathrm{e}}(x)\right\}<\infty$. Finally, note that $\psi_{+}(u)$ satisfies the defective renewal equation (3.1). Thus, by Lemma 4.1 and (4.3), we obtain

$$
\psi_{+}(u) \sim \frac{1+\theta}{\theta} z(u) \sim \frac{1}{\theta} \bar{F}_{\mathrm{e}}(u),
$$

which yields (4.1).

Remark 4.1. For the classical ruin probability $\phi(u)$, if $F_{\mathrm{e}} \in \delta$ then

$$
\phi(u) \sim \frac{1}{\theta} \bar{F}_{\mathrm{e}}(u) .
$$

Hence, Theorem 4.1 shows that for heavy-tailed claims, the absolute ruin probability and the classical ruin probability are asymptomatically equal, namely

$$
\psi_{+}(u) \sim \phi(u)
$$

This result confirms that large or heavy-tailed claims are very dangerous in the sense that large claims will cause ruin and absolute ruin with the same probability when the initial surplus is sufficiently large. Furthermore, the result implies that if a heavy-tailed claim is large enough to cause classical ruin then it is large enough to cause absolute ruin as well.

We note that there is one additional condition for (4.1) as compared with (4.5). The additional condition of $r(x) \rightarrow 0$ as $x \rightarrow \infty$ is not very restrictive. Many interesting heavytailed distributions satisfy the conditions of Theorem 4.1 such as Pareto, Burr, and log-normal distributions. More such distributions can be found in Embrechts et al. (1999). However, we point out that the main restriction of the condition is that the distribution has to be continuous.

In addition, many sufficient conditions for $F_{\mathrm{e}} \in \delta$ are given in terms of $r(x)$. For example, Theorem 3 of Embrechts and Villasenor (1988) means that if $\lim _{x \rightarrow \infty} r(x)=0$ and $\lim _{x \rightarrow \infty} x^{2} r^{\prime}(x)=-\infty$, then $F_{\mathrm{e}}(x) \in$ s. Hence, one corollary of Theorem 4.1 is that if $\lim _{x \rightarrow \infty} r(x)=0$ and $\lim _{x \rightarrow \infty} x^{2} r^{\prime}(x)=-\infty$, then (4.6) holds. Many other sufficient conditions for $F_{\mathrm{e}} \in \&$ can be found in Embrechts et al. (1999).

Next, we consider the asymptotical behavior of the absolute ruin probability for light-tailed claims. A claim size distribution is said to be light tailed if the moment generating function of the distribution exists. 
Theorem 4.2. Assume that there exists a positive constant $\kappa$ such that $\int_{0}^{\infty} \mathrm{e}^{\kappa x} \mathrm{~d} F_{\mathrm{e}}(x)=1+\theta$ and $\int_{0}^{\infty} \mathrm{e}^{\kappa x} \bar{F}_{\mathrm{e}}(x) \mathrm{d} x<\infty$. Then we have

$$
\psi_{+}(u) \sim C \phi(u)
$$

for a constant $C$ with $0<C<1$.

Proof. Under the conditions of Theorem 4.2, using the Lundberg-Cramér asymptotical formula for the classical ruin probability, we obtain

$$
\phi(u) \sim \frac{\int_{0}^{\infty} \mathrm{e}^{\kappa x} \bar{F}_{\mathrm{e}}(x) \mathrm{d} x}{\int_{0}^{\infty} x \mathrm{e}^{\kappa x} \mathrm{~d} F_{\mathrm{e}}(x)} \mathrm{e}^{-\kappa x} .
$$

Furthermore, we know that $\psi_{+}(u)$ satisfies the defective renewal equation (3.1) and the function $z(x)$ in (3.3) for $\psi_{+}(u)$ is reduced to

$$
z(x)=\frac{\lambda}{c} \int_{x}^{\infty}\left(\int_{t}^{t+c / \delta} \psi_{-}(t-y) \mathrm{d} F(y)+\bar{F}\left(t+\frac{c}{\delta}\right)\right) \mathrm{d} t .
$$

It is easy to see from $0<\psi_{-}(u)<1$ that

$$
0<z(x)<\frac{\lambda}{c} \int_{x}^{\infty}\left(F\left(t+\frac{c}{\delta}\right)-F(t)+\bar{F}\left(t+\frac{c}{\delta}\right)\right) \mathrm{d} t=\left(\frac{1}{1+\theta}\right) \bar{F}_{\mathrm{e}}(x),
$$

which implies that $\int_{0}^{\infty} \mathrm{e}^{\kappa x} z(x) \mathrm{d} x<\infty$ since $\int_{0}^{\infty} \mathrm{e}^{\kappa x} \bar{F}_{\mathrm{e}}(x) \mathrm{d} x<\infty$. Then, by Proposition 3.11.1 of Resnick (1992), we obtain

$$
\psi_{+}(u) \sim \frac{(1+\theta) \int_{0}^{\infty} \mathrm{e}^{\kappa x} z(x) \mathrm{d} x}{\int_{0}^{\infty} x \mathrm{e}^{\kappa x} \mathrm{~d} F_{\mathrm{e}}(x)} \mathrm{e}^{-\kappa x}
$$

Hence, (4.8) and (4.10) imply

$$
\psi_{+}(u) \sim \frac{(1+\theta) \int_{0}^{\infty} \mathrm{e}^{\kappa x} z(x) \mathrm{d} x}{\int_{0}^{\infty} \mathrm{e}^{\kappa x} \bar{F}_{\mathrm{e}}(x) \mathrm{d} x} \phi(u),
$$

which, together with (4.9), implies that there exists a constant

$$
C=\frac{(1+\theta) \int_{0}^{\infty} \mathrm{e}^{\kappa x} z(x) \mathrm{d} x}{\int_{0}^{\infty} \mathrm{e}^{\kappa x} \bar{F}_{\mathrm{e}}(x) \mathrm{d} x}
$$

such that $0<C<1$ and (4.7) holds.

It is well known that the positive constant $\kappa$ is unique provided it exists. However, there exist distributions that satisfy $\int_{0}^{\infty} \mathrm{e}^{\kappa x} \mathrm{~d} F_{\mathrm{e}}(x)<1+\theta$ for any $\kappa>0$. See, for example, Embrechts et al. (1999, p. 33) for details. Theorems 4.1 and 4.2 show that the relationship between the absolute ruin probability and the classical ruin probability for small claims are different from that for large claims. 


\section{Explicit results for exponential claims}

In this section, we assume that $F(y)=1-\mathrm{e}^{-y / \mu}, y \geq 0$, is an exponential distribution function with mean $\mu$. In this case, (2.14) is reduced to

$$
(\lambda+\alpha) \Phi_{-}(u)-(\delta u+c) \Phi_{-}^{\prime}(u)-\lambda A(u)=\frac{\lambda}{\mu} \mathrm{e}^{-u / \mu} \int_{-c / \delta}^{u} \mathrm{e}^{x / \mu} \Phi_{-}(x) \mathrm{d} x .
$$

Differentiating (5.1) with respect to $u$ and then rearranging, we obtain

$$
\left(a_{2} u+b_{2}\right) \Phi_{-}^{\prime \prime}(u)+\left(a_{1} u+b_{1}\right) \Phi_{-}^{\prime}(u)+b_{0} \Phi_{-}(u)=-\lambda\left(A^{\prime}(u)+\frac{1}{\mu} A(u)\right),
$$

where

$$
a_{2}=\delta, \quad b_{2}=c, \quad a_{1}=\frac{\delta}{\mu}, \quad b_{1}=\frac{c}{\mu}+\delta-\lambda-\alpha, \quad b_{0}=-\frac{\alpha}{\mu} .
$$

For $\alpha=0$, we can obtain the explicit expression for $\Phi_{-}(u)$ by solving the differential equation (5.2) and then obtain the explicit expression for $\Phi_{+}(u)$ using (3.2).

Theorem 5.1. Let $\alpha=0$ and let $F$ be an exponential distribution with mean $\mu$. If the function $A(x)$ defined in (2.3) satisfies (2.7) then, for any $-c / \delta<u<0$, we obtain

$$
\Phi_{-}(u)=C_{1}-\int_{u}^{0} \mathrm{e}^{-x / \mu}(\delta x+c)^{-1+\lambda / \delta}\left[C_{2}+\int_{0}^{x} \mathrm{e}^{y / \mu}(\delta y+c)^{1-\lambda / \delta} g(y) \mathrm{d} y\right] \mathrm{d} x,
$$

where the function $g(u)$ is defined as

$$
g(u)=-\frac{\lambda\left(\mu A^{\prime}(u)+A(u)\right)}{\mu(\delta u+c)} .
$$

Also, the constants $C_{1}$ and $C_{2}$ are given by

$$
\begin{aligned}
C_{1} & =\frac{-\lambda \beta_{3} P(-c / \delta)+\lambda \beta_{2}[Q(-c / \delta)+A(-c / \delta)]}{\lambda \beta_{2}+\left(c-\lambda \beta_{1}\right) P(-c / \delta)}, \\
C_{2} & =\frac{-\lambda \beta_{3}-\left(c-\lambda \beta_{1}\right)[Q(-c / \delta)+A(-c / \delta)]}{\lambda \beta_{2}+\left(c-\lambda \beta_{1}\right) P(-c / \delta)},
\end{aligned}
$$

where

$$
\begin{aligned}
\beta_{1} & =\mu\left(1-\mathrm{e}^{-c /(\delta \mu)}\right) \\
\beta_{2} & =\mu\left[-P(-c / \delta) \mathrm{e}^{-c /(\delta \mu)}+\frac{c^{\lambda / \delta}}{\lambda}\right] \\
\beta_{3} & =\int_{-c / \delta}^{0} \mathrm{e}^{x / \mu} Q(x) \mathrm{d} x-\int_{0}^{\infty} A(t) \mathrm{d} t \\
P(u) & =\int_{u}^{0} \mathrm{e}^{-x / \mu}(\delta x+c)^{-1+\lambda / \delta} \mathrm{d} x \\
Q(u) & =\int_{u}^{0} \mathrm{e}^{-x / \mu}(\delta x+c)^{-1+\lambda / \delta}\left(\int_{0}^{x} \mathrm{e}^{y / \mu}(\delta y+c)^{1-\lambda / \delta} g(y) \mathrm{d} y\right) \mathrm{d} x .
\end{aligned}
$$


Proof. When $\alpha=0,(5.2)$ is reduced to

$$
\Phi_{-}^{\prime \prime}(u)+f(u) \Phi_{-}^{\prime}(u)=g(u),
$$

where $f(u)=(\delta u+c+\mu(\delta-\lambda)) /(\mu(\delta u+c))$. The general solution to (5.8) is

$$
\begin{aligned}
\Phi_{-}(u) & =C_{1}+\int \exp \left(-\int f(u) \mathrm{d} u\right)\left(C_{2}+\int \exp \left(\int f(u) \mathrm{d} u\right) g(u) \mathrm{d} u\right) \mathrm{d} u \\
& =C_{1}-C_{2} P(u)-Q(u),
\end{aligned}
$$

where $C_{1}$ and $C_{2}$ are arbitrary constants. See, for example, Polyanin and Zaitsev (1995, p. 212). Thus, by (2.15) and (5.9), we obtain

$$
\int_{0}^{\infty} B(t) \mathrm{d} t=C_{1} \beta_{1}-C_{2} \beta_{2}-\beta_{3} .
$$

Furthermore, by (2.6) and (3.6), we obtain

$$
\Phi_{-}(0-)=\frac{\lambda}{c} \int_{0}^{\infty} B(t) \mathrm{d} t,
$$

which, together with (5.9), (5.10), $P(0)=0$, and $Q(0)=0$, implies that

$$
C_{1}=\frac{\lambda}{c}\left(C_{1} \beta_{1}-C_{2} \beta_{2}-\beta_{3}\right) .
$$

Moreover, if (2.7) holds, then (2.8) and (5.9) yield

$$
C_{1}-C_{2} P\left(-\frac{c}{\delta}\right)-Q\left(-\frac{c}{\delta}\right)=A\left(-\frac{c}{\delta}\right) .
$$

Therefore, solving (5.12) and (5.13), we obtain (5.5) and (5.6).

We point out that if $A(x)$ satisfies (2.9), then we can also obtain the explicit expression for $\Phi_{-}(u)$ by replacing $(5.13)$ by $C_{1}-C_{2} P(-c / \delta)-Q(-c / \delta)=0$. We omit the expression for $\Phi_{-}(u)$ in this case since most intersecting penalty functions satisfy (2.7).

Next, we illustrate the applications of Theorem 5.1 by giving explicit expressions for the absolute ruin probability and the (defective) distribution function of the deficit at absolute ruin when absolute ruin occurs in the following examples.

Example 5.1. (Absolute ruin probability.) When $\alpha=0$ and $w\left(x_{1}, x_{2}\right)=1$, we obtain $A(u)=$ $\mathrm{e}^{-u / \mu} \mathrm{e}^{-c /(\delta \mu)}, A(-c / \delta)=1, \Phi_{-}(u)=\psi_{-}(u)$, and $\Phi_{+}(u)=\psi_{+}(u)$. It is easy to verify that the function defined in (5.4) satisfies $g(u)=0$. Hence, the function defined in (5.7) satisfies $Q(u)=0$ and $\beta_{3}=-\mu \mathrm{e}^{-c /(\delta \mu)}$. Thus, (5.5) and (5.6) give

$$
\begin{gathered}
C_{1}=\frac{c^{\lambda / \delta}}{c^{\lambda / \delta}+\lambda \theta P(-c / \delta)}, \\
C_{2}=\frac{-\lambda \theta}{c^{\lambda / \delta}+\lambda \theta P(-c / \delta)},
\end{gathered}
$$

which, together with (5.3), imply that the absolute ruin probability satisfies

$$
\psi_{-}(u)=\frac{1+(\lambda \theta / c) \int_{u}^{0} \mathrm{e}^{-x / \mu}(1+\delta x / c)^{-1+\lambda / \delta} \mathrm{d} x}{1+(\lambda \theta / c) \int_{-c / \delta}^{0} \mathrm{e}^{-x / \mu}(1+\delta x / c)^{-1+\lambda / \delta} \mathrm{d} x}
$$

for $-c / \delta<u<0$. 
Equation (5.16) yields Equation (3.12) of Dassios and Embrechts (1989). Furthermore, by (2.15) and (5.11) and noting that $\bar{F}(x)=\bar{F}_{\mathrm{e}}(x)=\mathrm{e}^{-x / \mu}$ for $x \geq 0$, we have

$$
\begin{aligned}
\int_{x}^{\infty} B(t) \mathrm{d} t & =\int_{x}^{\infty} \int_{t}^{t+c / \delta} \psi_{-}(t-y) \frac{1}{\mu} \mathrm{e}^{-y / \mu} \mathrm{d} y \mathrm{~d} t+\int_{x}^{\infty} A(t) \mathrm{d} t \\
& =\left(\int_{0}^{\infty} B(t) \mathrm{d} t\right) \mathrm{e}^{-x / \mu} \\
& =\frac{c}{\lambda} \psi_{-}(0-) \bar{F}_{\mathrm{e}}(x)
\end{aligned}
$$

for $x \geq 0$, which implies that the function defined in (3.3) satisfies $z(x)=\psi_{-}(0-) \bar{F}_{\mathrm{e}}(x)$. It follows from (5.16) that

$$
\psi_{-}(0-)=\left(1+\frac{\lambda \theta}{c} \int_{-c / \delta}^{0} \mathrm{e}^{-x / \mu}\left(1+\frac{\delta x}{c}\right)^{-1+\lambda / \delta} \mathrm{d} x\right)^{-1} .
$$

Hence, for $u \geq 0,(3.2)$ and (3.7) yield

$$
\psi_{+}(u)=\psi_{-}(0-) \frac{1+\theta}{\theta} \int_{0}^{u} \bar{F}_{\mathrm{e}}(u-x) \mathrm{d} G(x)=(1+\theta) \psi_{-}(0-) \phi(u) .
$$

It is well known that when $F$ is the exponential distribution, the classical ruin probability $\phi(u)$ is given by

$$
\phi(u)=\frac{1}{1+\theta} \exp \left(-\frac{\lambda \theta}{c} u\right), \quad u \geq 0 .
$$

Then, by (5.16) and (5.17), we obtain that the absolute ruin probability satisfies

$$
\psi_{+}(u)=\frac{\exp (-(\lambda \theta / c) u)}{1+(\lambda \theta / c) \int_{-c / \delta}^{0} \exp (-x / \mu)(1+\delta x / c)^{-1+\lambda / \delta} \mathrm{d} x}
$$

for $u \geq 0$, which recovers Theorem 12 of Dassios and Embrechts (1989).

Example 5.2. (The deficit at absolute ruin.) When $\alpha=0$ and $w\left(x_{1}, x_{2}\right)=\mathbf{1}_{\left\{x_{2} \leq y\right\}}$ for $y \geq c / \delta$, we obtain $A(u)=\mathrm{e}^{-u / \mu}\left(\mathrm{e}^{-c /(\delta \mu)}-\mathrm{e}^{-y / \mu}\right), A\left(-\frac{c}{\delta}\right)=1-\mathrm{e}^{c /(\delta \mu)} \mathrm{e}^{-y / \mu}$, and

$$
\Phi(u)=\mathrm{P}\left(\left|U_{\delta}\left(T_{\delta}\right)\right| \leq y, T_{\delta}<\infty \mid U_{\delta}(0)=u\right)=G(y, u),
$$

which is the (defective) distribution function of the deficit at absolute ruin when absolute ruin occurs. It is easy to see that $A(u)$ satisfies (2.7) and the function defined in (5.4) satisfies $g(u)=0$. Hence, the function defined in (5.7) satisfies $Q(u)=0$ and $\beta_{3}=-\mu\left(\mathrm{e}^{-c /(\delta \mu)}-\mathrm{e}^{-y / \mu}\right)$. Thus, (5.5) and (5.6) give

$$
\begin{aligned}
& C_{1}=\frac{c^{\lambda / \delta}\left(1-\mathrm{e}^{c /(\delta \mu)} \mathrm{e}^{-y / \mu}\right)}{c^{\lambda / \delta}+\lambda \theta P(-c / \delta)}, \\
& C_{2}=\frac{-\lambda \theta\left(1-\mathrm{e}^{c /(\delta \mu)} \mathrm{e}^{-y / \mu}\right)}{c^{\lambda / \delta}+\lambda \theta P(-c / \delta)} .
\end{aligned}
$$

Notice that the function $g(u)=0$ in (5.3) and the constants in (5.14) and (5.15) are proportional to those in (5.18) and (5.19) respectively, by the same factor $1-\mathrm{e}^{c /(\delta \mu)} \mathrm{e}^{-y / \mu}$. 
Thus, by (5.3), for $-c / \delta<u<0$ and $y \geq c / \delta$, we obtain

$$
\begin{aligned}
G(y, u) & =\psi_{-}(u)\left(1-\mathrm{e}^{c /(\delta \mu)} \mathrm{e}^{-y / \mu}\right) \\
& =\frac{1+(\lambda \theta / c) \int_{u}^{0} \mathrm{e}^{-x / \mu}(1+\delta x / c)^{-1+\lambda / \delta} \mathrm{d} x}{1+(\lambda \theta / c) \int_{-c / \delta}^{0} \mathrm{e}^{-x / \mu}(1+\delta x / c)^{-1+\lambda / \delta} \mathrm{d} x}\left(1-\mathrm{e}^{c /(\delta \mu)} \mathrm{e}^{-y / \mu}\right) .
\end{aligned}
$$

Furthermore, by (2.15) and (5.11) and noting that

$$
\int_{x}^{\infty} A(t) \mathrm{d} t=\mu \mathrm{e}^{-x / \mu}\left(\mathrm{e}^{-c /(\delta \mu)}-\mathrm{e}^{-y / \mu}\right)
$$

and $\bar{F}(x)=\bar{F}_{\mathrm{e}}(x)=\mathrm{e}^{-x / \mu}$ for $x \geq 0$, we have

$$
\begin{aligned}
\int_{x}^{\infty} B(t) \mathrm{d} t & =\int_{x}^{\infty} \int_{t}^{t+c / \delta} \Phi_{-}(t-y) \frac{1}{\mu} \mathrm{e}^{-y / \mu} \mathrm{d} y \mathrm{~d} t+\int_{x}^{\infty} A(t) \mathrm{d} t \\
& =\left(\int_{0}^{\infty} B(t) \mathrm{d} t\right) \mathrm{e}^{-x / \mu}=\frac{c}{\lambda} \Phi_{-}(0-) \bar{F}_{\mathrm{e}}(x)
\end{aligned}
$$

for $x \geq 0$, which implies that the function defined in (3.3) satisfies $z(x)=\Phi_{-}(0-) \bar{F}_{\mathrm{e}}(x)=$ $G(y, 0-) \bar{F}_{\mathrm{e}}(x)$ for $y \geq c / \delta$ and $x \geq 0$. It follows from (5.20) that, for $y \geq c / \delta$,

$$
G(y, 0-)=\frac{1-\mathrm{e}^{c /(\delta \mu)} \mathrm{e}^{-y / \mu}}{1+(\lambda \theta / c) \int_{-c / \delta}^{0} \mathrm{e}^{-x / \mu}(1+\delta x / c)^{-1+\lambda / \delta} \mathrm{d} x} .
$$

Hence, for $u \geq 0$ and $y \geq c / \delta,(3.2)$ and (3.7) yield

$$
\begin{aligned}
G(y, u) & =G(y, 0-) \frac{1+\theta}{\theta} \int_{0}^{u} \bar{F}_{\mathrm{e}}(u-x) \mathrm{d} G(x) \\
& =(1+\theta) G(y, 0-) \phi(u) \\
& =\frac{\left(1-\mathrm{e}^{c /(\delta \mu)} \mathrm{e}^{-y / \mu}\right) \exp \left(-\frac{\lambda \theta}{c} u\right)}{1+(\lambda \theta / c) \int_{-c / \delta}^{0} \mathrm{e}^{-x / \mu}(1+\delta x / c)^{-1+\lambda / \delta} \mathrm{d} x} .
\end{aligned}
$$

Therefore, the conditional distribution function of the deficit at absolute ruin, given that absolute ruin occurs, satisfies

$$
\mathrm{P}\left(\left|U_{\delta}\left(T_{\delta}\right)\right| \leq y \mid T_{\delta}<\infty\right)=\frac{G(y, u)}{\psi_{+}(u)}=\left(1-\mathrm{e}^{c /(\delta \mu)} \mathrm{e}^{-y / \mu}\right)
$$

for $u \geq 0$ and $y \geq c / \delta$. Thus, the expected deficit at absolute ruin, given that absolute ruin occurs, satisfies

$$
\mathrm{E}\left(\left|U_{\delta}\left(T_{\delta}\right)\right| \mid T_{\delta}<\infty\right)=\frac{1}{\mu} \int_{c / \delta}^{\infty} y \mathrm{e}^{c /(\delta \mu)} \mathrm{e}^{-y / \mu} \mathrm{d} y=\mu+\frac{c}{\delta}
$$

for $u \geq 0$. Equation (5.21) means that when the claim size distribution $F$ is the exponential distribution,

$$
\mathrm{E}\left(\left|U_{\delta}\left(T_{\delta}\right)\right| \mid T_{\delta}<\infty\right)=\mathrm{E}(|U(T)| \mid T<\infty)+\frac{c}{\delta},
$$

since $\mathrm{E}(|U(T)| \mid T<\infty)=\mu$ in this case. However, we do not know if (5.22) holds for general claim size distributions. 
Similarly, we can obtain explicit expressions for the distribution functions of the surplus just before absolute ruin and the amount causing absolute ruin when absolute ruin occurs by, respectively, setting $w\left(x_{1}, x_{2}\right)=\mathbf{1}_{\left\{x_{1} \leq x\right\}}$, for $x>-c / \delta$, and $w\left(x_{1}, x_{2}\right)=\mathbf{1}_{\left\{x_{1}+x_{2} \leq y\right\}}$, for $y>0$, in Theorem 5.1. More results related to absolute ruin can also be obtained by considering suitable penalty functions.

When $\alpha>0$, the general solutions to the second-order differential equation (5.2) are available but more complicated. We illustrate the solutions to (5.2) by deriving explicit expressions for the Laplace transform of the time to absolute ruin.

Example 5.3. (The Laplace transform of the time to absolute ruin.) When $w\left(x_{1}, x_{2}\right)=1$, we obtain $A(u)=\mathrm{e}^{-u / \mu} \mathrm{e}^{-c / \delta \mu}, A(-c / \delta)=1$, and $\Phi(u)=\mathrm{E}\left(\mathrm{e}^{-\alpha T_{\delta}} \mathbf{1}_{\left\{T_{\delta}<\infty\right\}}\right)$ is the Laplace transform of the time to absolute ruin. It is easy to see that $A(u)$ satisfies (2.7). Thus, it follows from (2.8) that

$$
\lim _{u \downarrow-c / \delta} \Phi_{-}(u)=\frac{\lambda}{\lambda+\alpha} .
$$

Furthermore, it is easy to verify that $A^{\prime}(u)+(1 / \mu) A(u)=0$. Thus, by the transforms $\Phi_{-}(u)=$ $y(x)$ and $x=\left(u+b_{2} / a_{2}\right) /\left(-a_{2} / a_{1}\right)=-\mu(u+c / \delta)$, for $-c / \delta<u<0,(5.2)$ is reduced to a confluent hypergeometric equation

$$
x y_{x x}^{\prime \prime}+(b-x) y_{x}^{\prime}-a y=0, \quad x<0,
$$

where

$$
b=\frac{a_{2} b_{1}-a_{1} b_{2}}{a_{2}^{2}}=1-\frac{\lambda+\alpha}{\delta} \text { and } a=\frac{b_{0}}{a_{1}}=-\frac{\alpha}{\delta} .
$$

The general solution to (5.24) is a linear combination of two independent solutions. Hence, by Equations (13.1.15) and (13.1.18) of Abramowitz and Stegun (1972), we obtain

$$
y(x)=c_{1} \mathrm{e}^{x} U(b-a, b ;-x)+c_{2}(-x)^{1-b} \mathrm{e}^{x} M(1-a, 2-b ;-x), \quad x<0,
$$

where $M(a, b ; x)$ and $U(a, b ; x)$ are the confluent hypergeometric functions of the first and second kinds respectively, and $c_{1}$ and $c_{2}$ are arbitrary constants. Thus,

$$
\Phi_{-}(u)=y\left(-\mu\left(u+\frac{c}{\delta}\right)\right)=c_{1} h_{1}(u)+c_{2} h_{2}(u), \quad-c / \delta<u<0,
$$

where

$$
\begin{aligned}
& h_{1}(u)=\mathrm{e}^{-\mu(u+c / \delta)} U\left(b-a, b ; \mu\left(u+\frac{c}{\delta}\right)\right), \\
& h_{2}(u)=\left(\mu\left(u+\frac{c}{\delta}\right)\right)^{1-b} \mathrm{e}^{-\mu(u+c / \delta)} M\left(1-a, 2-b ; \mu\left(u+\frac{c}{\delta}\right)\right) .
\end{aligned}
$$

By (2.13) and using arguments similar to those used for (5.2), we can show that $\Phi_{+}(u)$ satisfies the following second-order differential equation:

$$
c \Phi_{+}^{\prime \prime}(u)+\left(\frac{c}{\mu}-\lambda-\alpha\right) \Phi_{+}^{\prime}(u)-\frac{\alpha}{\mu} \Phi_{+}(u)=-\lambda\left(B^{\prime}(u)+\frac{1}{\mu} B(u)\right), \quad u \geq 0 .
$$

When $F$ is the exponential distribution, it is easy to verify from (2.15) that

$$
B^{\prime}(u)+\frac{1}{\mu} B(u)=A^{\prime}(u)+\frac{1}{\mu} A(u)=0 .
$$


Hence, (5.26) is reduced to

$$
\Phi_{+}^{\prime \prime}(u)+p \Phi_{+}^{\prime}(u)+q \Phi_{+}(u)=0, \quad u \geq 0,
$$

where

$$
p=\frac{1}{\mu}-\frac{\lambda+\alpha}{c} \text { and } q=-\frac{\alpha}{\mu c}
$$

Because $p^{2}-4 q>0$, the general solution to (5.27) is

$$
\Phi_{+}(u)=d_{1} \exp \left\{\frac{\sqrt{p^{2}-4 q}-p}{2} u\right\}+d_{2} \exp \left\{-\frac{\sqrt{p^{2}-4 q}+p}{2} u\right\}, \quad u \geq 0,
$$

where $d_{1}$ and $d_{2}$ are arbitrary constants. However, $\lim _{u \rightarrow \infty} \Phi_{+}(u)=0$. Hence, $d_{1}$ must be zero. Thus,

$$
\Phi_{+}(u)=d_{2} \exp \left\{-\frac{p+\sqrt{p^{2}-4 q}}{2} u\right\}, \quad u \geq 0 .
$$

To specify the expressions of $\Phi_{+}(u)$ and $\Phi_{-}(u)$, we need to determine the constants $c_{1}, c_{2}$, and $d_{2}$. In doing so, by Equations (13.5.10) and (13.5.12) of Abramowitz and Stegun (1972), we know that if $b \neq 0$ then $\lim _{u \downarrow-c / \delta} h_{1}(u)=\Gamma(1-b) / \Gamma(1-a)$, where $\Gamma(\cdot)$ is the gamma function. Thus, by (2.6), (2.16), (5.23), and $\lim _{u \downarrow-c / \delta} h_{2}(-c / \delta)=0$, we obtain the following three equations:

$$
\begin{aligned}
c_{1} h_{1}(0)+c_{2} h_{2}(0) & =d_{2}, \\
c_{1} h_{1}^{\prime}(0)+c_{2} h_{2}^{\prime}(0) & =-\frac{d_{2}}{2}\left(p+\sqrt{p^{2}-4 q}\right), \\
c_{1} \frac{\Gamma(1-b)}{\Gamma(1-a)} & =\frac{\lambda}{\lambda+\alpha} .
\end{aligned}
$$

Solving the three equations we determine the required constants as follows:

$$
\begin{aligned}
c_{1} & =\frac{\lambda \Gamma(1-a)}{(\lambda+\alpha) \Gamma(1-b)}, \\
c_{2} & =\frac{-\lambda \Gamma(1-a)\left(h_{1}^{\prime}(0)+\left(\left(p+\sqrt{p^{2}-4 q}\right) / 2\right) h_{1}(0)\right)}{(\lambda+\alpha) \Gamma(1-b)\left(h_{2}^{\prime}(0)+\left(\left(p+\sqrt{p^{2}-4 q}\right) / 2\right) h_{2}(0)\right)}, \\
d_{2} & =\frac{\lambda \Gamma(1-a)\left(h_{1}(0) h_{2}^{\prime}(0)-h_{1}^{\prime}(0) h_{2}(0)\right)}{(\lambda+\alpha) \Gamma(1-b)\left(h_{2}^{\prime}(0)+\left(\left(p+\sqrt{p^{2}-4 q}\right) / 2\right) h_{2}(0)\right)} .
\end{aligned}
$$

Using

$$
\frac{\mathrm{d}}{\mathrm{d} x} M(a, b ; x)=\frac{a}{b} M(a+1, b+1 ; x) \quad \text { and } \quad \frac{\mathrm{d}}{\mathrm{d} x} U(a, b ; x)=-a U(a+1, b+1 ; x),
$$


we obtain explicit expressions for $h_{1}(0), h_{1}^{\prime}(0), h_{2}(0)$, and $h_{2}^{\prime}(0)$ as follows:

$$
\begin{aligned}
& h_{1}(0)= \mathrm{e}^{-\mu c / \delta} U\left(b-a, b ; \frac{\mu c}{\delta}\right), \\
& h_{1}^{\prime}(0)=-\mu \mathrm{e}^{-\mu c / \delta}\left[U\left(b-a, b ; \frac{\mu c}{\delta}\right)+(b-a) U\left(b-a+1, b+1 ; \frac{\mu c}{\delta}\right)\right], \\
& h_{2}(0)=\left(\frac{\mu c}{\delta}\right)^{1-b} \mathrm{e}^{-\mu c / \delta} M\left(1-a, 2-b ; \frac{\mu c}{\delta}\right), \\
& h_{2}^{\prime}(0)=\mu\left(\frac{\mu c}{\delta}\right)^{1-b} \mathrm{e}^{-\mu c / \delta}\left[\left((1-b)\left(\frac{\mu c}{\delta}\right)^{-1}-1\right) M\left(1-a, 2-b ; \frac{\mu c}{\delta}\right)\right. \\
&\left.+\frac{1-a}{2-b} M\left(2-a, 3-b ; \frac{\mu c}{\delta}\right)\right] .
\end{aligned}
$$

Hence, if $b \neq 0$ then the explicit expressions for the Laplace transform of the time to absolute ruin are given by (5.28) and (5.25) for $u \geq 0$ and $-c / \delta<u<0$, respectively, where the constants $c_{1}, c_{2}$, and $d_{2}$ are specified by (5.30)-(5.36).

Finally, we point out that if $b=0$, then Equation (13.5.11) of Abramowitz and Stegun (1972) yields $\lim _{u \downarrow-c / \delta} h_{1}(u)=1 / \Gamma(1+a)$. The explicit expressions for the Laplace transforms are still given by (5.28) and (5.25) for $u \geq 0$ and $-c / \delta<u<0$, respectively. However, the constants $c_{1}, c_{2}$, and $d_{2}$ are specified by replacing (5.29) by $c_{1} / \Gamma(1+a)=\lambda /(\lambda+\alpha)$ and solving the corresponding three equations. We point out that the case of $b=0$ or $\delta=\lambda+\alpha$ is less important in practice since the interest force $\delta$ is usually less than one while the Poisson rate $\lambda$ is usually larger than one. Therefore, we omit the expressions for the constants $c_{1}, c_{2}$, and $d_{2}$ in this case.

\section{Conclusions}

We assume that an insurer could borrow money at a debit interest rate when the insurer is on deficit. With debit interest, absolute ruin may occur, namely, the surplus of the insurer is no longer able to return to a positive level. It is interesting both in theory and in application to consider absolute ruin questions and the effect of debit interest on the surplus of an insurer. As shown in this paper, many absolute ruin questions can be studied in a unified approach by the expected discounted penalty function. We expect that this approach can be employed to study absolute ruin questions in other risk models. However, there are still some interesting questions on this topic that cannot be dealt with by the expected discounted penalty function, such as the joint distribution of the absolute ruin time and the classical ruin time, the distribution of the total duration of the negative surplus until absolute ruin, and so on. We leave these issues for further research.

\section{Acknowledgements}

This research was supported by the Natural Sciences and Engineering Research Council of Canada (NSERC) under grant RGPIN250031-06. The author would like to thank an anonymous referee for a careful reading of the paper and detailed comments that improved the presentation of the paper. 


\section{References}

Abramowitz, M. and Stegun, I. A. (1972). Handbook of Mathematical Functions: with Formulas, Graphs, and Mathematical Tables. US Government Printing Office, Washington, DC.

Asmussen, S. (2000). Ruin Probabilities. World Scientific, Singapore.

Bingham, N. H., Goldie, C. M. and Teugels, J. L. (1987). Regular Variation. Cambridge University Press.

CAI, J. (2004). Ruin probabilities and penalty functions with stochastic rates of interest. Stoch. Process. Appl. 112, 53-78.

CAI, J. And Dickson, D. C. M. (2002). On the expected discounted penalty function at ruin of a surplus process with interest. Insurance Math. Econom. 30, 389-404.

CAI, J. AND TANG, Q. (2004). On max-sum-equivalence and convolution closure of heavy-tailed distributions and their applications. J. Appl. Prob. 41, 117-130.

Dassios, A. AND Embrechts, P. (1989). Martingales and insurance risk. Stoch. Models 5, 181-217.

Dickson, D. C. M. AND Egídio dos Reis, A. D. (1997). The effect of interest on negative surplus. Insurance Math. Econom. 21, 1-16.

Embrechts, P. ANd Schmidli, H. (1994). Ruin estimation for a general insurance risk model. Adv. Appl. Prob. 26, 404-422.

EMbrechts, P. And Villasenor, J. A. (1988). Ruin estimates for large claims. Insurance Math. Econom. 7, $269-274$.

Embrechts, P., KlüPpelberg, C. And Mikosch, T. (1999). Modelling Extremal Events for Insurance and Finance. Springer, New York.

Gerber, H. U. And ShiU, E. S. W. (1997). The joint distribution of the time of ruin, the surplus immediately before ruin, and the deficit at ruin. Insurance Math. Econom. 21, 129-137.

Gerber, H. U. And Shiu, E. S. W. (1998). On the time value of ruin. N. Amer. Actuarial J. 2, 48-78.

Polyanin, A. D. And Zaitsev, V. F. (1995). Handbook of Exact Solutions for Ordinary Differential Equations. CRC Press, New York.

RESNICK, S. I. (1992). Adventures in Stochastic Processes. Birkhäuser, Boston, MA.

Sundt, B. ANd Teugels, J. L. (1995). Ruin estimates under interest force. Insurance Math. Econom. 16, 7-22.

Yin, C. C. AND ZHAO, J. S. (2006). Nonexponential asymptotics for the solutions of renewal equations, with applications. J. Appl. Prob. 43, 815-824. 